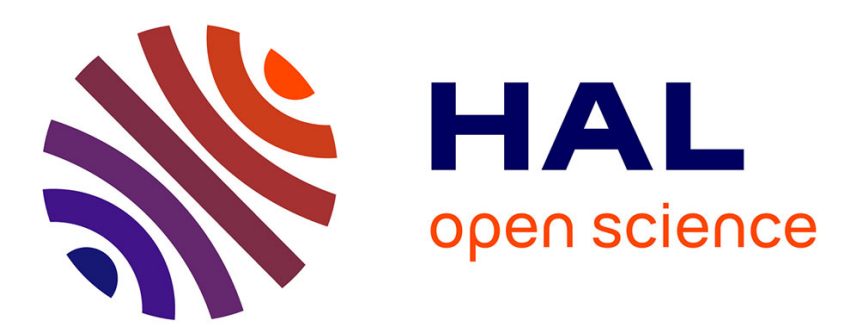

\title{
Enhancement of energy harvesting using acoustical-black-hole-inspired wave traps
}

Fabien Maugan, Simon Chesné, Mélodie Monteil, Manuel Collet, Kaijun Yi

\section{To cite this version:}

Fabien Maugan, Simon Chesné, Mélodie Monteil, Manuel Collet, Kaijun Yi. Enhancement of energy harvesting using acoustical-black-hole-inspired wave traps. Smart Materials and Structures, 2019, 10.1088/1361-665X/ab1f11 . hal-02132429

\section{HAL Id: hal-02132429 \\ https://hal.science/hal-02132429}

Submitted on 18 Sep 2020

HAL is a multi-disciplinary open access archive for the deposit and dissemination of scientific research documents, whether they are published or not. The documents may come from teaching and research institutions in France or abroad, or from public or private research centers.
L'archive ouverte pluridisciplinaire HAL, est destinée au dépôt et à la diffusion de documents scientifiques de niveau recherche, publiés ou non, émanant des établissements d'enseignement et de recherche français ou étrangers, des laboratoires publics ou privés. 


\title{
Enhancement of energy harvesting using acoustical-black-hole-inspired wave traps
}

\author{
Fabien Maugan ${ }^{1}$, Simon Chesne ${ }^{1}\left(10\right.$, Melodie Monteil ${ }^{1}$, Manuel Collet $^{2}$ and \\ Kaijun $\mathbf{Y i}^{2}$ \\ ${ }^{1}$ Univ. Lyon, CNRS INSA-Lyon, LaMCoS UMR5259, F-69621 Villeurbanne, France \\ ${ }^{2}$ LTDS UMR5513 Ecole Centrale de Lyon, 36 Avenue Guy de Collongue, F-69130 Ecully, France \\ E-mail: simon.chesne@insa-lyon.fr
}

\begin{abstract}
The purpose of this paper is to highlight the interest of associating smart functionalities in the same smart-structure. It consists in combining a wave trap device with an energy harvester on a beam. Both functions are performed using piezoelectric transducers. The wave trap device is based on the acoustical black hole concept. It is designed to generate a gradual modification of the equivalent composite Young's modulus along the beam. The aim is to concentrate the mechanical energy at the beam center where the harvesting device is placed. To achieve this, a network of transducers is used. The transducers are shunted on a specific negative capacitance. The harvester device is made of a simple piezoelectric patch associated with a classical extracting circuit. A significant increase in harvested energy is observed using this association for both weak and strong coupling. From the practical viewpoint, the study also highlights the sensitivity of the structural behavior, which strongly depends on the tuning of the trap device.
\end{abstract}

Keywords: energy harvesting, piezoelectric patches, piezoelectric shunt, structural vibrations

\section{Introduction}

Over recent decades, technological innovations have been developed to efficiently decrease structural masses. This exploit, which is very useful in industrial application in many domains, has been made possible by new machining processes able to produce stiff but light architectures, and by increasingly advanced numerical optimization routines. The emergence of new materials such as composites has helped this trend.

These improvements have converged with, as a side effect, increased sensitivity to dynamical excitation. Several original fields of research such as metacomposites and structronics are being investigated in this context to explore how to modify the dynamical properties of systems [1]. The targeted applications concern structural stability, acoustic attenuation, health monitoring, and energy harvesting [2] to ensure the robustness and autonomy of active subsystems. Recent studies have shown the potential of sound energy harvesting using acoustic metastructures [3-5].
One of the most important challenges in harvesting energy is to improve and increase the amount of mechanical energy in the specific domain in which the harvester operates. Although much research focuses on the enhancement of the harvester itself through passive and active processes [6, 7], many publications have addressed the modification of vibrating structures alone. The idea of focusing wave energy at a specific point of a structure has already been employed in [8-11] where, for instance, elliptical and parabolic acoustical mirrors are used to focus vibration energy on the harvesting system. References [12-14] can also be cited with improvements based on the addition of a nonlinear circuit, the integration of metamaterials and the use of photonic crystals, respectively. The study presented here treats the case of an academic structure equipped with a piezoelectrical transducer network that forms a wave trap device. The principle of this method has already shown its potential in [12, 15, 16]. It works in a way very similar to the black hole technology presented in [17], but in this case, the material properties of the structure are modified by the piezoelectric patch network 


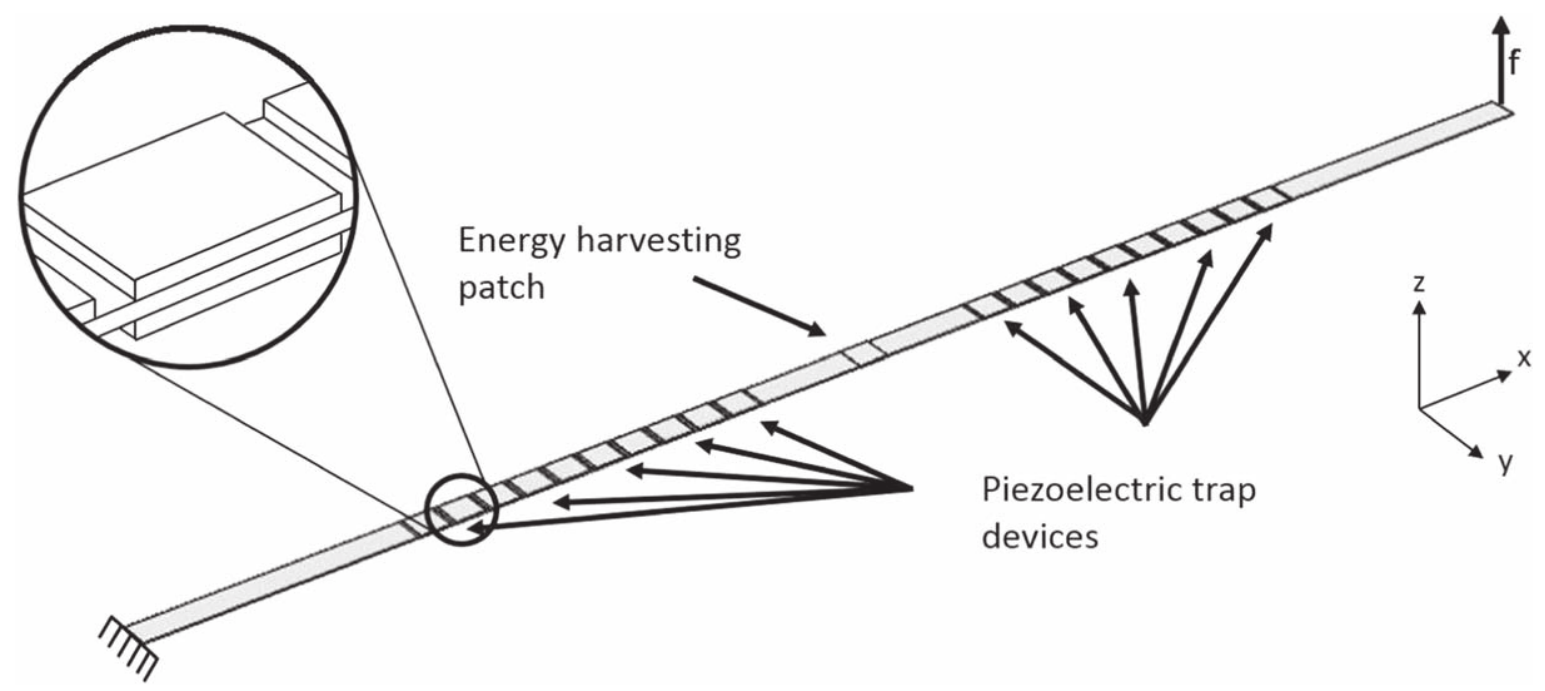

Figure 1. The beam studied with the piezoelectric trap device and piezoelectric harvester.

that gradually softens the composite structure so as to concentrate the energy.

These years, so called acoustical black holes have been the subject of many interesting developments. By tailoring the structure itself $[18,19]$ or by using shunted transducers as piezoelectric patches in order to created adaptive gradientindex metamaterials [20]. These structures can have many objectives as vibration damping [21,22] or energy harvesting as investigated in this present study.

In these studies, the harvesting devices are simply modeled by resistive circuits to focus our analysis on the mechanical part of the system. Although this approximation can be useful in an initial academic approach, it is not representative of a real-life harvester that has to convert AC to DC to provide useful energy to the power electronics components. This model also masks certain instability problems arising from the nonlinear components of the harvesting device. Consequently, a standard DC harvester is used in this work.

The purpose of this paper is to highlight the interest of using a piezoelectric trap device to improve energy harvesting from a mechanical vibrating structure. After the presentation of the innovative smart structure of interest and its trap device and harvesting technology, the study focuses on the theoretical background of energy harvesting with piezoelectric patches. In the third part, the numerical application of the hypothesis of constant displacement is dealt with and the benefits of using the trap device are explained. The extension of the results to the hypothesis of constant force and its effects are discussed in the final part.

\section{Piezoelectric trap device for energy harvesting}

The proposed smart structure concept is illustrated by an aluminum cantilever beam. It comprises a piezoelectric trap device concentrating the mechanical energy in a specific subdomain, where a harvesting system is located. This section describes these three different parts of the system.
Table 1. First 5 structural $z$ bending modes with relevant coupling coefficients.

\begin{tabular}{lcc}
\hline $\begin{array}{l}\text { Mode } \\
\text { number }\end{array}$ & $\begin{array}{c}\text { Frequency } \\
\mathrm{Hz}\end{array}$ & $\begin{array}{c}\text { Coupling } \\
\text { factor }\end{array}$ \\
\hline 7 & 34.683 & 0.0023 \\
11 & 65.212 & 0.0046 \\
14 & 102.75 & 0.0078 \\
17 & 151.46 & 0.0122 \\
20 & 209.74 & 0.0162 \\
\hline
\end{tabular}

\subsection{Structure of interest}

The mechanical system of interest consists of a $2.5 \mathrm{~m}$ cantilever beam $3 \mathrm{~mm}$ thick and $5 \mathrm{~cm}$ wide. 40 piezoelectric patches are installed on the beam to build a trap device. Twenty of them are placed on each side of the central zone, called the trap zone, which is $40 \mathrm{~cm}$ long. The patches are glued in pairs on both of these sides, 10 on the top and 10 on the bottom of the beam. The beam is shown in figure 1. The patches are electrically shunted with a specific negative capacitance (NC) circuit, making the whole network a wave trap device. The last patch is added at the center of the beam and connected to the harvesting circuit. The beam is fixed-free and the excitation is ensured at its free end.

The energy harvesting efficiency of the patches is directly correlated to the effective piezoelectric coupling coefficient. Table 1 shows the electromechanical coupling factor value for the first five bending modes of the structure in the $z$ direction. Its value has been obtained using a Commercial FE Tool by comparing the system eigenfrequencies with its patches short and open circuited. This factor can be considered as the ratio between the converted energy during the quasi-static loading phase and the maximum energy storable in the piezoelectric component. Its design can be found in [23]. Firstly, it is easy to understand that the $y$ bending and the torsion modes always lead to very low coupling factors for such networks. Regarding the $z$ bending modes, two conditions must 


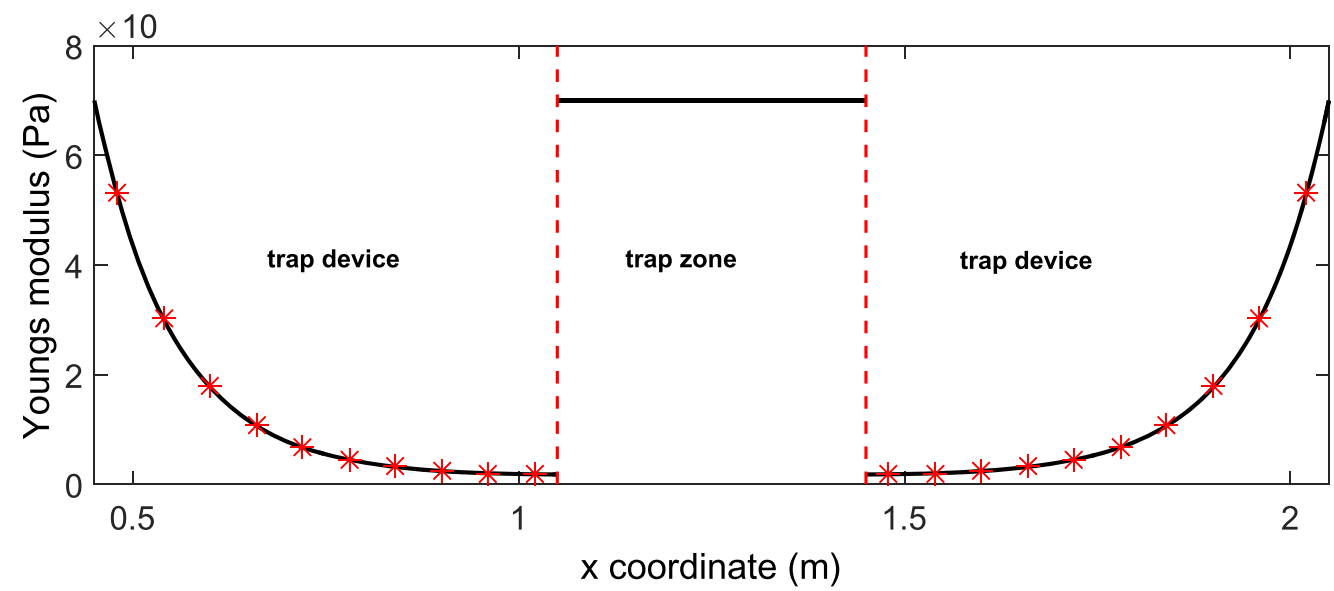

Figure 2. Discrete softening effect provided by the trap device (red stars) compared to the reference continuous profile (solid black line).
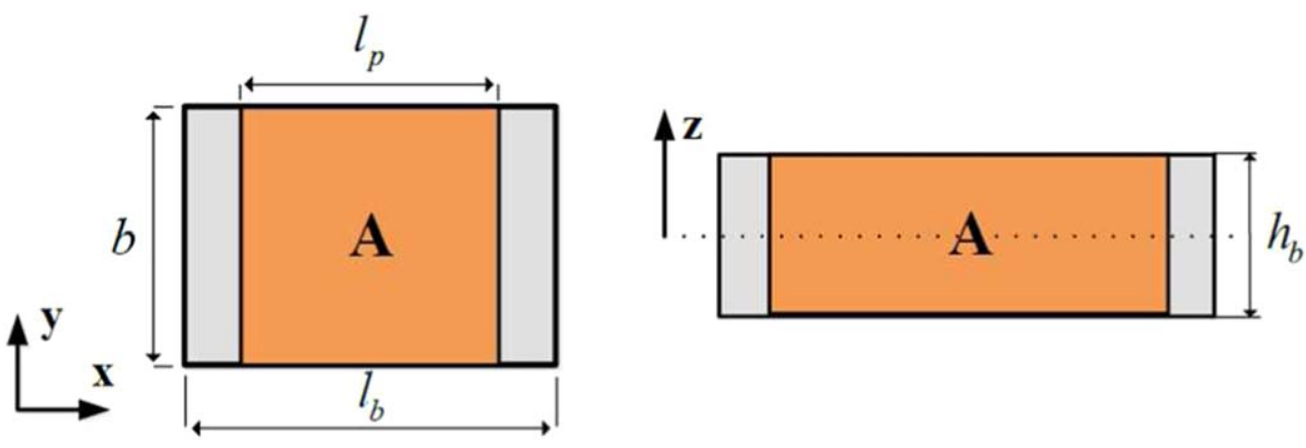

Figure 3. Geometrical properties of the unit cell.

converge to obtain a large coupling factor. First, the observed mode has to be high enough in rank to efficiently strain the patches. In addition, the patch must be on a vibration antinode to be appropriate. Finally, all these conditions are satisfied only by modes $7,14,17$ and 20, whose frequency and coupling factors are given in table 1 . For all the other modes, these two conditions are not satisfied and the resulting coupling factor is always lower than $10^{-3}$.

\subsection{Trap device design}

The trap devices described above can be built in many different ways. They can be obtained by designing one of the material parameters or geometry parameters or even a combination of these parameters in the trap device zones, so as to obtain a black hole effect as described in [16, 17]. In this work, a reference trap device was designed by gradually modifying the effective Young's modulus along the beam, as shown in figure 2.

The principles of this concept was established in [18]. According to this theory, the Young's modulus evolution of the trap $E_{\text {trap }}$ is designed as a function of the longitudinal coordinate $x$ shown in equation (1) where $E_{b}=6.9 e 10 \mathrm{~Pa}$ and sech is the hyperbolic secant function:

$$
E_{\text {trap }}=E_{b} * \operatorname{sech}(0.6 * x) \text {. }
$$

Table 2. Geometrical characteristics of the unit cell.

\begin{tabular}{cccc}
\hline$l_{b}$ & $l_{p}$ & $\mathrm{~b}$ & $h_{b}$ \\
\hline $0.06 \mathrm{~m}$ & $0.05 \mathrm{~m}$ & $0.05 \mathrm{~m}$ & $3 e-3 \mathrm{~m}$ \\
\hline
\end{tabular}

Note that this pattern has not to be optimal. Theoretical studies on acoustic black holes have shown that a smooth gradient is sufficient to generate the desired effect.

Only the portion of the beam with the trap device is presented in figure 2 (between 0.4 and $2.1 \mathrm{~m}$ ). The Young modulus outside this portion is equal to $69 \mathrm{GPa}$. The material's stiffness steadily decreased to concentrate the mechanical energy in the beam center where the harvesting device is located. This reference continuous trap device is purely theoretical and current technology is unable to produce such a structure with sufficient accuracy. This drawback is overcome by discretizing the evolution of the Young's modulus.

The reference trap device discretization must be divided into a large enough number of portions to reduce as much as possible the wave reflection on each of the portion interfaces. These considerations lead to a division of each side into ten equal elements corresponding to each pair of patches on both beam sides. A unit cell is shown in figure 3 and the corresponding geometry parameters are given in table 2. Each cell has its own target Young's modulus, symbolized by the 

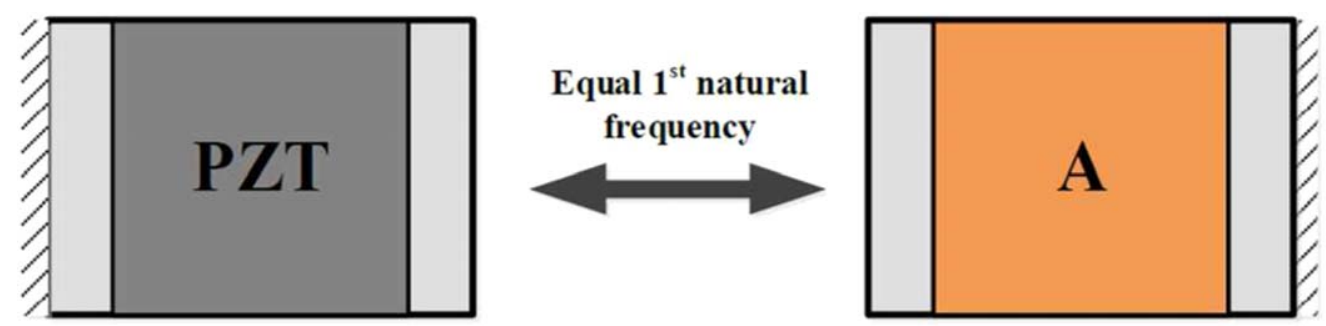

Figure 4. Equivalence between a piezoelectrical cantilever and a unit cell.

Table 3. Trap device negative capacitance values.

\begin{tabular}{llllllllll}
\hline \multicolumn{10}{c}{ Negative capacitance values $\left({ }^{*} 1 e-7 \mathrm{~F}\right)$} \\
\hline-1.1148 & -1.0395 & -1.0253 & -1.0203 & -1.0181 & -1.0171 & -1.0164 & -1.0162 & -1.016 & -1.016 \\
\hline
\end{tabular}

red stars in figure 2. The used methodology is precisely presented in [15].

As mentioned previously, the final trap device is composed of a piezoelectric patch network designed to mimic the reference trap device. This performance is obtained by a NC shunt capable of softening the structure locally. Using an active electrical circuit to reduce the capacitance value of the piezoelectric component, which directly affects the electromechanical coupling factor and thus the conversion performance of the device, was first proposed by Forward [24] in 1979. This concept is now an important source of publications in research [25-27] and applications [28-32].

Two equivalent finite element models of each unit cell (see figure 3) were built in order to correctly tune the $\mathrm{NC}$ shunts and obtain the targeted Young's modulus described in figure 2. The first is a fixed-free unit cell with a homogeneous Young's modulus whose first eigenfrequency is $f_{1}$. This model represents the target composite structure discretized by unit cell. The second is a fixed-free unit cell made of aluminum with one piezoelectric patch on both sides. Each patch is shunted with a variable NC. This model represents the reallife structure and must be tuned.

The optimal NC value for each unit cell is that giving the same first eigenfrequency $f_{1}$. This equivalence is schematized in figure 4. The NC values determined for one half of the trap can be found in table 3 . Obviously this approach has some limitations linked to the size of the cells. One is the discretisation of the Young's modulus profile (figure 2), which moves away the resulting structure from the ideal one. The size of the cell is also a limitation at high frequency. The calculus leading to the tuning of the shunts is valid only if the considered wavelength is larger than the size of the cell. Which is the case in the following of the study, where the behavior of the structure correspond to the desired one.

\subsection{Harvesting circuit}

The harvesting system used in this paper is a very standard circuit composed of a diode bridge, a smoothing capacitor $C_{R}$ and a resistor $R$ which represent the electronic system to be powered. It is connected to a transducer represented by a current source $i_{1}$ and a piezoelectric capacitance $C_{0}$. The whole system is connected to the piezoelectric element located at the center of the cantilever beam. The diode breakdown voltage is $V_{D}=1 e-8 \mathrm{~V}$ which can be neglected in relation to the other voltages. The $R C$ time constant value is $\tau=R C_{R}=10 \mathrm{~s}$ to guarantee that steady state is reached within a reasonable amount of time.

There are other more complicated and optimized harvesting electronics. For example, parallel and series SSHI [33] include an additional switch mounted in parallel or in series in the harvesting loop. This switch allows treating the piezoelectric voltage nonlinearly and increasing the harvested power. These more complex extraction techniques associated with a trap device will be addressed in further studies.

\section{Electromechanical model}

The aim of this section is to present the theoretical background of the standard harvesting device proposed by [34] and to express the output voltage and the harvested power which are the figures of interest of this paper. These expressions are given in both constant displacement and constant force hypotheses to represent different levels of coupling between the mechanical and the electronic parts of the system.

For the sake of clarity, a simple but representative one degree of freedom mechanical model is used. Although the global operation of the system is modified by the trap device in the following, the harvesting principle remains the same, and the conclusions obtained remain true.

\subsection{Multimodal modeling of smart structures}

The constitutive equations of piezoelectricity were standardized by the IEEE in 1988 [35]. It defines these components as an electromechanical coupling which modifies the basic motion equation such that:

$$
\left\{\begin{array}{c}
M \ddot{u}+C \dot{u}+K_{E} u+\alpha V=F \\
\alpha \dot{u}-C_{0} \dot{V}=I
\end{array},\right.
$$




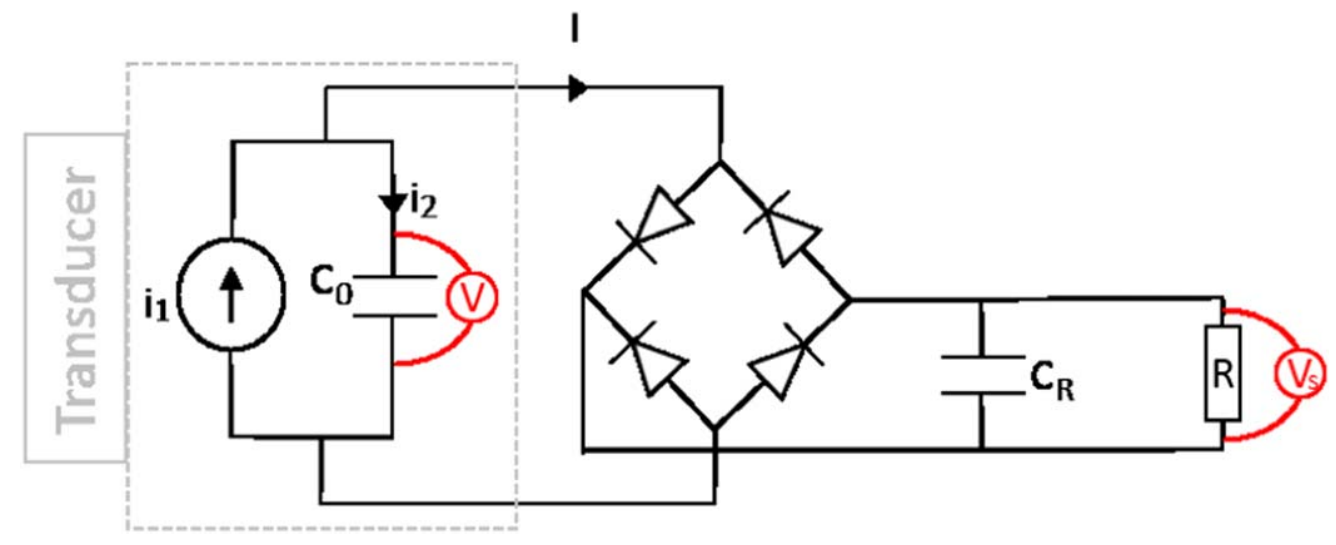

Figure 5. Harvesting circuit and its associated transducer.

where the scalars $M, C$ and $K_{E}$ represent the dynamical mass, structural damping and the stiffness, respectively, in the short-circuited case. $\alpha$ is the equivalent force factor or coupling term. Finally, $V$ and $I$ are the voltage and the outgoing current of the transducer.

In the case presented, and in accordance with [36], the system is excited with a sinus force $F$ with a constant module $F_{m}$ :

$$
F=F_{m} \sin \left(\omega_{m} t\right) .
$$

It is reasonable to expect $u$ to have the same form:

$$
u=u_{m} \sin \left(\omega_{m} t+\varphi\right),
$$

where $\omega_{m}$ represents one system eigenpulsation. The latter depends on the coupling and varies as a function of the connected electrical impedance $Z$ as long as the impedance is linear. It can be determined through the system simulation by searching for the exact value where the force and velocity signals are phased.

The energy balance is obtained by multiplying equation (2) by $\dot{u}$ and integrating:

$$
\left\{\begin{array}{c}
\int F \ddot{u} d t=\frac{1}{2} M \dot{u}^{2}+\frac{1}{2} K_{E} u^{2}+\int C \dot{u}^{2} d t+\int \alpha V \dot{u} d t \\
\int \alpha V \dot{u} d t=\frac{1}{2} C_{0} V^{2}+\int V I d t
\end{array} .\right.
$$

In the case where only one mode is considered, the assumption $u=\Phi q$ with $\Phi$ being the first modal shape obtained at 0 voltage and $q$ the modal coordinate, is possible. The modal projection of the system can then be described as follows:

$$
\left\{\begin{array}{c}
m \ddot{u}+c \dot{u}+k_{E} u+\theta V=f \\
\theta^{T} \dot{q}-C_{0} \dot{V}=I
\end{array}\right.
$$

with $m=\Phi^{T} M \Phi, c=\Phi^{T} C \Phi, k_{E}=\Phi^{T} K_{E} \Phi, f=\Phi^{T} F$, and $\theta=\Phi^{T} \alpha$.

The whole theory attached to piezoelectric physics and the electromechanical coupled system can easily be found in the literature. A particularly detailed and complete example is contained in [23] but will not be described further in this paper. The demonstration focuses here on the two figures of merit which are the harvested power $P$ and the output voltage, symbolized as $V_{s}$ in figure 5 .

\subsection{Energy harvesting with piezoelectric patches: weak and strong couplings}

$P$ is the power through resistor $R$ which symbolized an electronic device to be supplied:

$$
P=\frac{V_{s}^{2}}{R} .
$$

As the current in $C_{R}$ (see figure 5) is null [33] the current can be formalized as follows:

$$
\int_{0}^{\frac{T_{0}}{2}} I d t=\frac{V_{s}}{R} \frac{T_{0}}{2}
$$

With $T_{0}=2 \pi / \omega_{0}$. The second line of the equation (2) becomes:

$$
\begin{aligned}
\int_{0}^{\frac{T_{0}}{2} I d t} & =\theta^{T} \int_{0}^{\frac{T_{0}}{2}} \dot{q} d t-C_{0} \int_{0}^{\frac{T_{0}}{2}} \dot{V} d t \\
& =\theta^{T}\left(q\left(t_{2}\right)-q\left(t_{1}\right)\right)-C_{0}\left(V\left(t_{2}\right)-V\left(t_{1}\right)\right) \\
& =2 \theta^{T} q_{m}-2 C_{0} V_{s} \\
& =\frac{V_{s}}{R} \frac{T_{0}}{2} .
\end{aligned}
$$

By combining equations (8) and (9) the expression of the output voltage can finally be written as:

$$
V_{s}=\frac{R \theta}{C_{0} R \omega_{m}+\frac{\pi}{2}} \omega_{m} q_{m}
$$

Considering the energy balance on the same half period with no kinetics or potential energy variation, equation (6) leads to:

$$
\int_{0}^{T_{o} / 2} f \dot{q} d t=c \int_{0}^{T_{o} / 2} \dot{q}^{2} d t+V_{s} \int_{0}^{T_{o} / 2} I d t .
$$

Which can be developed using the resonance with 


$$
\begin{aligned}
& q=q_{m} \sin \left(\omega_{0} t+\frac{\pi}{2}\right): \\
& \int_{0}^{T_{o} / 2} f \dot{q} d t=f_{m} q_{m} \omega_{0} \int_{0}^{T_{o} / 2} \sin ^{2}\left(\omega_{0} t\right) d t \\
& =f_{m} q_{m} \omega_{0} \int_{0}^{T_{o} / 2}\left(\frac{1-\cos \left(2 \omega_{0} t\right)}{2}\right) d t \\
& =\frac{f_{m} q_{m} \omega_{0}}{2} \frac{T_{0}}{2} \\
& =\frac{f_{m} q_{m} \pi}{2}, \\
& c \int_{0}^{T_{o} / 2} \dot{q}^{2} d t=c q_{m}^{2} \omega_{0}^{2} \int_{0}^{T_{o} / 2} \sin ^{2}\left(\omega_{0} t\right) d t \\
& =\frac{c q_{m}^{2} \omega_{0} \pi}{2} \text {. }
\end{aligned}
$$

Recombining equations (8), (12) and (13) gives the maximal amplitude of the modal coordinate:

$$
q_{m}=\frac{f_{m}}{c \omega_{0}+{\frac{2 R \omega_{0} \theta}{\left(R C_{0} \omega_{0}+\frac{\pi}{2}\right)}}^{2}} .
$$

Including (14) in (10), the complete expression for the theoretical voltage is:

$$
V_{s}^{t h}=\frac{R \theta}{C_{0} R \omega_{0}+\frac{\pi}{2}} \omega_{0} \frac{f_{m}}{c \omega_{0}+\frac{2 R \omega_{0} \theta}{\left(R C_{0} \omega_{0}+\frac{\pi}{2}\right)}} .
$$

This natural way of expressing the coupling between the mechanical system and the harvesting part entails working in the constant force framework. This ensures strong coupling in the whole system and the effect of the harvesting device on the global dynamics of the structure is taken into account. Considering a constant force model for complex structures leads to many computational difficulties due to nonlinear behaviors and can generate long or non-convergent procedures. These types of problems are addressed in this paper in section 4.3. In most publications, the results relating to energy harvesting using piezoelectric devices are given in the hypothesis of constant displacement, assuming that the $\alpha V$ is negligible in the first line of equation (5). This framework describes a system where the electronic effects of the harvesting device on the mechanical part can be neglected at the working frequencies. In other words, the harvesting device does not affect the global dynamics of the system. This is why this hypothesis is also described as a weak coupling, which suffices to represent the working conditions of a large number of applications. In this framework, the energy provided by the external force is now dissipated only through the structural damping, and equation (11) becomes:

$$
\int_{0}^{T_{o} / 2} f \dot{q} d t=c \int_{0}^{T_{o} / 2} \dot{q}^{2} d t
$$

The maximal amplitude of the modal coordinate is also modified:

$$
q_{m}=\frac{f_{m}}{c \omega_{m}} .
$$

Finally, the expression for the theoretical voltage is simplified by combining equations (17) and (10):

$$
V_{s}^{t h}=\frac{R \theta}{C_{0} R \omega_{0}+\frac{\pi}{2}} \frac{f_{m}}{c} .
$$

The constant displacement hypothesis leads to simple computations but the fact of ignoring the coupling must be validated to ensure the veracity of the results obtained. A comparison of the equations (15) and (18) shows that the constant force hypothesis adds damping in the output voltage of the system. This modification leads to a decrease in the harvested power but is better suited to the practical cases of working conditions. Indeed, the strong coupling hypothesis appears clearly more realistic for industrial applications for which the load can usually be associated with an external force and not with a displacement.

Figure 6 shows the comparison between the numerical and theoretical results for a one degree of freedom system (parameters are given in table 4) compliant with the previously defined structure of interest.

Curves in black solid lines are the direct translations of equations (15), (18) and (7). Time constant $\tau=R C_{R}$ is chosen as equal to 10 . The numerical results were computed differently depending on the coupling hypothesis. In the weak coupling case, the system is excited at $f=\sqrt{k_{E} / m}$ which corresponds exactly to the resonant frequency of the structure. Indeed, the weak coupling does not affect this parameter. In strong coupling cases, the voltage retro-action modifies the global dynamics. The input force signal must be tuned specifically for each resistance value with the speed signal to find the new resonance frequency.

The numerical and theoretical results match and allow validating the model.

\section{Numerical results}

The purpose of this section is to present the trap device effects on the structure's global dynamics and also to evaluate the improvement in energy harvesting due to the trap device activation in the classical framework of the hypothesis of constant displacements. Therefore, the constant force hypothesis is taken into account to estimate the energy loss due to the change of hypothesis.

Whereas the previous demonstrations were presented by considering only a one degree of freedom structure, all the forthcoming applications will be applied to the multimodal beam presented in section 2.1. Mechanical matrices, coupling coefficients and NCs values have been computed thanks to a Commercial FE tool. All the following steps have been made under Matlab and Simulink.

\subsection{Structural effect of the trap device}

The trap device is activated in this first application with all its NCs tuned to the values given in table 3 . The extension of the equation (2) to the multimodal case (equation (19)) allows taking into account the trap device's effects through the vector $\mathbf{C}_{n e g}$. Subscripts ${ }_{1-20}$ show the equation is written for a 

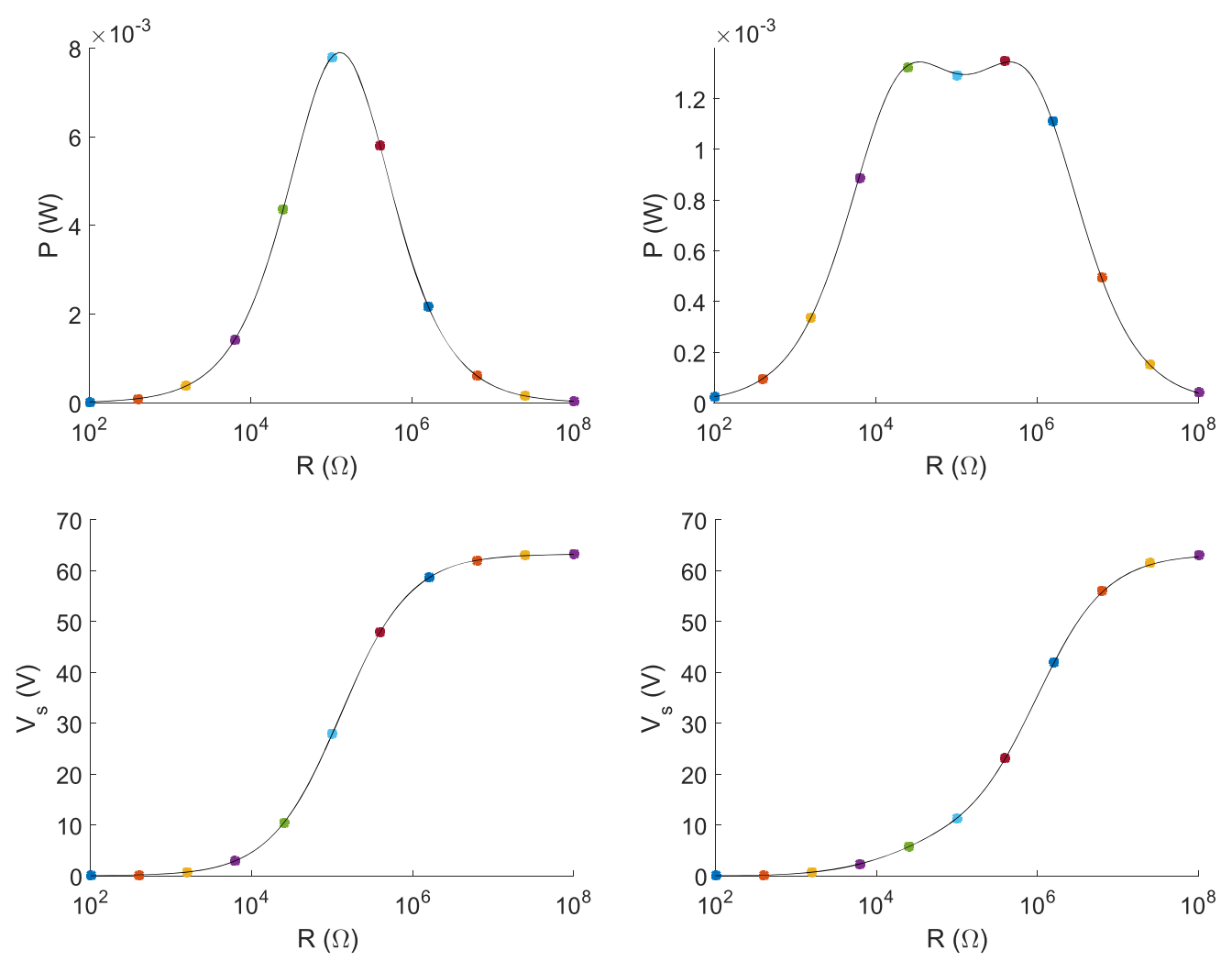

Figure 6. Voltage and harvested power for a monomodal model. Comparison between numerical and theoretical results for both weak (left) and strong (right) couplings.

Table 4. Parameter values for the one degree of freedom model.

\begin{tabular}{lc}
\hline Parameter & Value \\
Beam length & $250 \mathrm{~mm}$ \\
eigen-frequency & $35 \mathrm{~Hz}$ \\
\hline$f_{0}$ & $35.34 \mathrm{~Hz}$ \\
$f_{1}$ & $35.52 \mathrm{~Hz}$ \\
$\epsilon$ & 0.001 \\
$\lambda$ & $6.33 e-3 \mathrm{~V} \mathrm{~m}^{-1}$ \\
$C_{0}$ & $55.8 \mathrm{nF}^{-1}$ \\
$\alpha$ & $0.0035 \mathrm{~N} \mathrm{~V}^{-1}$ \\
$k_{E}$ & $2.17 e 4 \mathrm{~N} \mathrm{~m}^{-1}$ \\
$m$ & $440 \mathrm{~g}^{1}$ \\
$c$ & $0.216 \mathrm{~N} \mathrm{~m}^{1} \mathrm{~s}$ \\
$d t$ & $1 e-4 \mathrm{~s}$ \\
\hline
\end{tabular}

trap device with twenty pairs of patches. Let us define $\mathbf{I}_{\text {trap }}$ the first twenty terms of the current vector, $\boldsymbol{\theta}_{\text {trap }}^{T}$ the electromechanical coupling matrix of the twenty pairs of piezoelectric transducers, $\mathbf{C}_{01-20}$ the piezoelectric capacitances and $\mathbf{Q}$ the modal coordinates. $\boldsymbol{a}$ is a multiplying factor applied to the NC. Its value equals 1 for the optimal trap device designed previously. These values represent the ideal continuous case. The discretization of the trap and the coupling between cells slightly modify the dynamics of the device and the NC values must also be slightly modified

$$
\mathbf{I}_{\text {trap }}=a \mathbf{C}_{\text {neg }} \dot{\mathbf{V}}_{\text {trap }}=\boldsymbol{\theta}_{\text {trap }}^{T} \dot{\mathbf{Q}}-\mathbf{C}_{0 \text { trap }} \dot{\mathbf{V}}_{\text {trap }}
$$

The previous relation can be written in another way in order to obtain:

$$
\dot{\mathbf{V}}_{\text {trap }}=\frac{\boldsymbol{\theta}_{\text {trap }}^{T}}{a \mathbf{C}_{\text {neg }}+\mathbf{C}_{0 \text { trap }}} \dot{\mathbf{Q}} .
$$

Which allows deducing the modification in the structural stiffness due to the activation of the trap device:

$$
\tilde{\mathbf{k}}=\mathbf{k}+\boldsymbol{\theta}_{\text {trap }} \frac{1}{\boldsymbol{a}_{\text {neg }}+\mathbf{C}_{0 \text { trap }}} \boldsymbol{\theta}_{\text {trap }}^{T} \text {. }
$$

The motion equation (2) can finally be fully extended to the multimodal application case:

$$
\left\{\begin{array}{c}
\mathbf{m} \ddot{\mathbf{q}}+\boldsymbol{c} \dot{\mathbf{q}}+\tilde{\mathbf{k}} \mathbf{q}=\mathbf{f}-\boldsymbol{\theta}_{\text {har }} \mathbf{V}_{\text {har }} \\
\mathbf{I}_{h a r}=\boldsymbol{\theta}_{h a r}^{T} \dot{\boldsymbol{q}}-\mathbf{C}_{0 \text { har }} \dot{\mathbf{V}}_{h a r}
\end{array}\right.
$$

Let us assume $[16,37]$ that changes to mechanical property due to the trap device activation lead to changes in the structural response. The modified modal behavior can be attested by examining the evolution of the eigenfrequencies and eigenvectors due to the modification of the NCs.

Figure 7 displays the evolution of all the 20 first eigenfrequencies for a global multiplying factor $a$ between 0 and 2 applied to all the NCs. A factor 1 corresponds to the optimal theoretical value of $C_{n e g}$ computed using the values of table 3 . This figure allows determining different phenomena and behaviors as a function of the factor range:

- Unstable zone between $a=0.9$ and $a=1.05$,

- Stiffening of the system for $a$ lower than 1. Softening effect for $a$ higher than 1, 


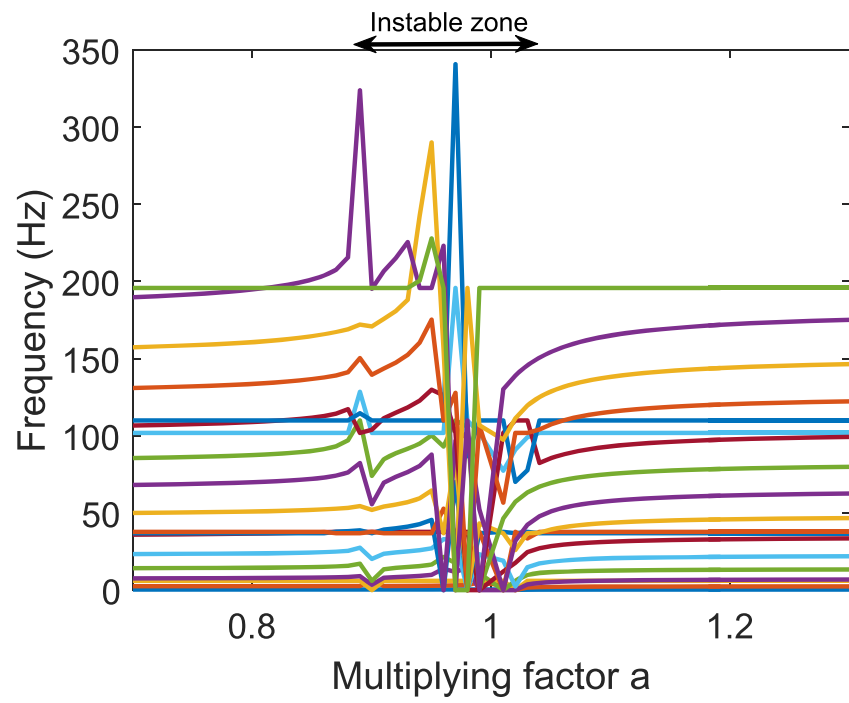

Figure 7. Eigenfrequency evolution for the first 20 structural modes depending on the coefficient $a$. One color corresponds to one structural mode.

- Eigenfrequencies tend to retrieve their nominal values outside the unstable zone.

- Several sweeping modes can be seen. Indeed, the trap effect is mainly effective on the $x$ bending modes.

As seen previously, the purpose of the trap device is to soften the structure as much as possible at its center in order to concentrate the larger part of the mechanical energy. This initial study gives the information that the optimal multiplying factor is close and higher than one.

A safety margin is necessary to avoid the unstable zone due to the network properties. Therefore, the next computation will be performed for $a=1.05$ as an optimal tuning of the trap device. This margin appears necessary because the computation of the $\mathrm{NC}$ values does not take into account the discretization effect of the trap device (presented in figure 2) or the coupling between each cell.

Figure 8 displays the MAC matrices obtained for the multiplying factors of 0.95 (a), 1.05 (b) and 2 (c). These figures reveal the trap device effect on the system eigenvectors and confirm that the system retrieves its nominal behavior when $a$ is far from 1. Non-null extra diagonal terms are due to switching modes.

All the MAC indexes are higher than 0.8. Although the trap device induces large changes in the eigenfrequency values, the associated deformed shapes are stable. From a more practical standpoint, if the harvesting device is optimally placed according to the nominal eigenvectors of the system, it remains efficient once the trap device is switched on.

All the modifications obtained by the trap device activation concentrate the mechanical energy at the point where the harvesting device is placed. The purpose of the next section is to highlight the improvement obtained for the energy harvesting due to the trap device activation.

\subsection{Constant displacement framework (weak coupling)}

Settings for the numerical application in the hypothesis of constant displacement have to be chosen wisely. As seen in table 1, only five modes can be targeted and produce energy, and only the 17th mode has a really significant coupling factor. This mode is the 12th bending mode and has a wavelength of $0.42 \mathrm{~m}$ which is 6 times the unit cell length. The question of the modal truncation at the 20th mode has been addressed and does not affect the results. Another important point consists of the modification of the global modal behavior of the structure due to the trap device activation. Before performing the computation and for the current NC setting, the exact frequency of the 17 th mode is computed and serves to solicit the structure to maximize its response. This setting is obtained using a MAC comparison with the nominal modal bases of the structure.

The results are displayed in figure 9 for twenty $R$ values. Due to the linear properties of the system, the harvested power is presented in a dimensionless units for the sake of representativity. The normalization is made according to the maximum harvested power under the constant displacement hypothesis and with the trap device activated. In this figure, the blue circles present the results without the trap device while the red crosses are obtained with the trap device activated. The increase due to the activation of the trap device is very clear for this figure of merit. This improvement is concentrated in the range $1 e 3 \Omega$ to $1 e 5 \Omega$ for the harvesting power, and can reach $68 \%$.

The obtained improvement can appear to be very high but it should be borne in mind that this computation is representative of a very specific case where the displacement is constant whatever the frequency, the applied load or the coupling. First, the coupling between the different piezoelectic devices and the structure are assumed to be perfect. In the real setup, the coupling between the structure and the transducer (see glue and positioning) is one of the critical points and directly affects the performances of both the trap device and the harvester. Here, the system is also assumed to be perfectly tuned in both the target mode frequency and the NC values (once the parameter $a$ chosen). The last point is that the trap device must be supplied with account taken of the amount of energy required.

Several issues had to be overcome to produce the previous results. Indeed, the diode bridge present in the electronic circuit of the harvesting device (see figure 5) induces numerous discontinuities in both the mechanical and the electrical signals. This leads to a very difficult convergence of the system to its steady state.

\subsection{Constant force framework (strong coupling)}

The previous results showed that the methodology adopted allowed obtaining reliable results in the case of the constant displacement hypothesis and for the cantilever smart beam. The effect of the trap device was identified in the system modal behavior and the nonlinear behavior of the electrical 

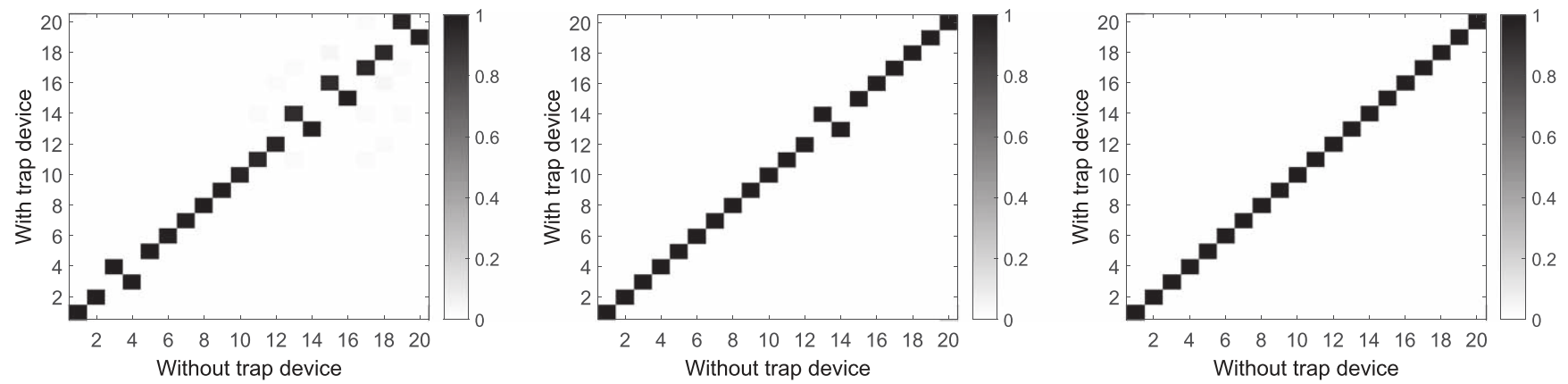

Figure 8. Mode shape evolution depending on $C_{n e g}$ coefficient multiplied by (a) 0.95 , (b) 1.05 , (c) 2 .

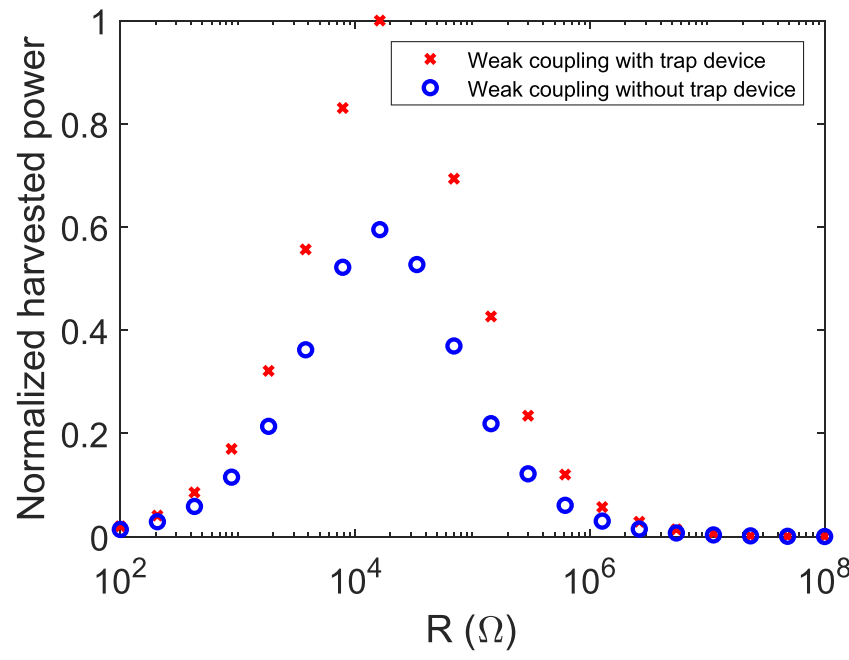

Figure 9. Harvested power in weak coupling with an multiplying coefficient of 1.05 applied to all the negative capacitances of the structure.

components was taken into account by using a more evolved solver.

The extension of this study to the constant force hypothesis case allowed considering the effect of the electrical circuit on the global dynamics of the structure. Coupling was modeled by connecting the output voltage harvesting device to the piezoelectric component. This looping induced another modification in the vibrating behavior of the whole system and the excitation device had to be tuned at the new frequency of interest. The search for the perfect tuning was performed in this application using an optimization loop included in the main procedure to find the excitation frequency giving the force and velocity signals in phase. Indeed, this condition is a very practical way of ensuring the system is tuned to its phase resonance which, in this case, is very close to the amplitude resonance. This tuning provides maximum harvested power.

Finally, the computation of the harvested power was performed on the whole frequency range in the constant force hypothesis case. Once again, in figure 10, the blue curves are obtained without trap device while the red ones are computed with the activated trap device.

Firstly, it can be seen that that, although the activation of the trap device still improves energy harvesting, the increase in this case is reduced to $37 \%$. Indeed, the new hypothesis

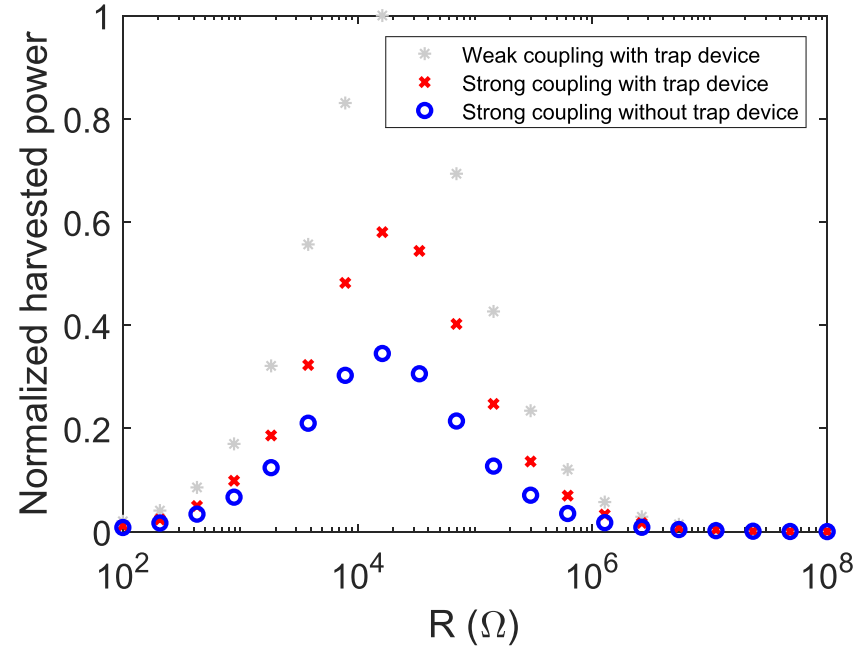

Figure 10. Harvested power in strong coupling with an multiplying coefficient of 1.05 applied to all the negative capacitances of the structure.

implies strong coupling between the electronic and mechanics fields, leading to a loss of part of the energy. This figures also allows comparing the last results to those obtained in the case of a constant displacement hypothesis with an activated trap device (curve in gray stars). The loss of power due to the strong coupling can be seen clearly in figure 10, proving that this hypothesis must be taken into account to better estimate the potential amount of harvested power.

This part of the study clearly shows the interest and importance of modeling the interactions and coupling between the different parts of the system. Although the constant force hypothesis is an important step in this direction, it implies considerable computation costs which are not always worth the trouble. Indeed, the value of the harvested power is not only very sensitive to the tuning of the system in frequency but also to its different NCs. The efficiency of the coupling between the patches and the structure can be another source of uncertainty. The results are consistent with the theoretical ones for the one degree of freedom system described in the previous sections. 


\section{Conclusion}

This study illustrated two main concepts: (i) the possibility of creating a synthetic wave trap, based on the acoustical black hole and using a discrete transducer network; and (ii) the interest of associating smart functionalities in the same smartstructure. A test structure was proposed to explore these concepts: it consisted of a beam combining a wave trap device with an energy harvester. The study used a multimodal model to select the adequate mode in view to illustrating these novelties. The wave trap was composed of 20 pairs of piezoelectric patches shunted with NC) The capacitances were adequately tuned to create a gradient in the resulting material property. The waves and thus the energy can be focused on the harvester by tuning the NC values of the cells composing the trap. The harvester device was composed of a simple piezoelectric patch associated with a classical extracting circuit. A significant increase of harvested energy was observed using this association for both weak and strong coupling. From the practical standpoint, the study also highlighted the sensitivity of the structural behavior (mode shapes and frequencies), which depended strongly on the tuning of the trap device. These complex aspects are often ignored in many studies and further studies are required to optimize them.

Since the NC shunts used to create the wave black hole are active elements, they need to be powered. It appears that it is also important to consider the energy balance of the harvesting system. Even if previous studies have shown, that the amount of consumed energy could be small compared with the harvested one [16, 37], this point needs to be clarified and will be addressed in future studies. Indeed, the presented structure used 20 pairs of piezoelectrical patches with an OPA and the energy balance does not allow to realize autonomous system. Nethertheless, mechanical energy relocation on the structure can be of a real interest in different configurations. For example it can be used to powered unconnected devices unreachable by RF lighting or other wireless power. It also remains a really interesting perspective. Experiments will be performed to validate and optimize the results presented in this paper and fully analyze the energy balance of the harvesting system.

\section{ORCID iDs}

Simon Chesne (iD https://orcid.org/0000-0003-1306-7231

\section{References}

[1] Collet M, Ouisse M and Tateo F 2014 Adaptive metacomposites for vibroacoustic control applications IEEE Sens. J. 14 2145-52

[2] Le M Q, Capsal J-F, Lallart M, Hebrard Y, Van Der Ham A, Reffe N, Geynet L and Cottinet P-J 2015 Review on energy harvesting for structural health monitoring in aeronautical applications Prog. Aerosp. Sci. 79 147-57
[3] Sun K H, Kim J E, Kim J and Song K 2017 Sound energy harvesting using a doubly coiled-up acoustic metamaterial cavity Smart Mater. Struct. 26075011

[4] Nguyen H, Zhu R, Chen J, Tracy S and Huang G 2018 Analytical coupled modeling of a magneto-based acoustic metamaterial harvester Smart Mater. Struct. 27055010

[5] Wang X, Xu J, Ding J, Zhao C and Huang Z 2019 A compact and low-frequency acoustic energy harvester using layered acoustic metamaterials Smart Mater. Struct. 28025035

[6] Jackson N, Stam F, Olszewski O Z, Doyle H, Quinn A and Mathewson A 2016 Widening the bandwidth of vibration energy harvesters using a liquid-based non-uniform load distribution Sensors Actuators A 246 170-9

[7] Zhu D, Tudor M J and Beeby S S 2010 Strategies for increasing the operating frequency range of vibration energy harvester: a review Meas. Sci. Technol. 2122001

[8] Carrara M, Cacan M, Leamy M, Ruzzene M and Erturk A 2012 Dramatic enhancement of structure-borne wave energy harvesting using an elliptical acoustic mirror Appl. Phys. Lett. 100204105

[9] Carrara M, Cacan M, Toussaint J, Leamy M, Ruzzene M and Erturk A 2013 Metamaterial-inspired structures and concepts for elastoacoustic wave energy harvesting Smart Mater. Struct. 22065004

[10] Carrara M, Kulpe J, Leadenham S, Leamy M and Erturk A 2015 Fourier transform-based design of a patterned piezoelectric energy harvester integrated with an elastoacoustic mirror Appl. Phys. Lett. 106013907

[11] Tol S, Degertekin F L and Erturk A 2016 Dramatic enhancement of elastic wave energy harvesting using a gradient-index phononic crystal lens ASME 2016 Conf. on Smart Materials, Adaptive Structures and Intelligent Systems (American Society of Mechanical Engineers) p V002T07A013

[12] Xiong L, Tang L and Mace B R 2016 Internal resonance with commensurability induced by an auxiliary oscillator for broadband energy harvesting Appl. Phys. Lett. 108203901

[13] Chen Z, Yang Y, Lu Z and Luo Y 2013 Broadband characteristics of vibration energy harvesting using onedimensional phononic piezoelectric cantilever beams Physica B 410 5-12

[14] Fan Y, Collet M, Ichchou M, Li L, Bareille O and Dimitrijevic Z 2016 A wave-based design of semi-active piezoelectric composites for broadband vibration control Smart Mater. Struct. 25055032

[15] Yi K, Collet M, Chesné S and Monteil M 2017 Enhancement of elastic wave energy harvesting using adaptive piezo-lens Mech. Syst. Sig. Process. 97 255-66

[16] Yi K, Monteil M, Collet M and Chesné S 2017 Smart metacomposite-based systems for transient elastic wave energy harvesting Smart Mater. Struct. 26035040

[17] Zhao L, Conlon S C and Semperlotti F 2014 Broadband energy harvesting using acoustic black hole structural tailoring Smart Mater. Struct. 23065021

[18] Vemula C, Norris A N and Cody G D 1996 Attenuation of waves in plates and bars using a graded impedance interface at edges J. Sound Vib. 196 107-27

[19] Nansha Gao N, Wei Z, Hou H and Krushynska A 2019 Design and experimental investigation of $\mathrm{v}$-folded beams with acoustic black hole indentations J. Acoust. Soc. Am. 145 79-83

[20] Chen Y Y, Zhu R, Barnhart V and Huang G L 2016 Enhanced flexural wave sensing by adaptive gradient-index metamaterials Sci. Rep. 635048

[21] McCormicka C and Shepherdb M R 2018 Optimal design and position of an embedded one-dimensional acoustic black hole Internoise 2018 (Chicago) pp 692-705 
[22] Zhou T and Cheng L 2018 A resonant beam damper tailored with acoustic black hole features for broadband vibration reduction J. Sound Vib. 430 174-84

[23] Preumont A 2006 Dynamics of Electromechanical and Piezoelectric Systems (Berlin: Springer) (https://doi.org/ 10.1007/1-4020-4696-0)

[24] Forward R L 1979 Electronic damping of vibrations in optical structures Appl. Opt. 18 690-7

[25] Date M, Kutani M and Sakai S 2000 Electrically controlled elasticity utilizing piezoelectric coupling J. Appl. Phys. 87 $863-8$

[26] Tang J and Wang K 2001 Active-passive hybrid piezoelectric networks for vibration control: comparisons and improvement Smart Mater. Struct. 10794

[27] Wu S-Y 2000 Broadband piezoelectric shunts for structural vibration control US Patent 6,075,309

[28] Behrens S, Fleming A and Moheimani S 2003 A broadband controller for shunt piezoelectric damping of structural vibration Smart Mater. Struct. 1218

[29] Bisegna P, Caruso G and Maceri F 2006 Optimized electric networks for vibration damping of piezoactuated beams J. Sound Vib. 289 908-37

[30] Kim J-S, Wang K and Smith E C 2005 High-authority piezoelectric actuation system synthesis through mechanical resonance and electrical tailoring J. Intell. Mater. Syst. Struct. 16 21-31
[31] Neubauer M, Oleskiewicz R, Popp K and Krzyzynski T 2006 Optimization of damping and absorbing performance of shunted piezo elements utilizing negative capacitance J. Sound Vib. 298 84-107

[32] Park C H and Baz A 2005 Vibration control of beams with negative capacitive shunting of interdigital electrode piezoceramics J. Vib. Control 11 331-46

[33] Badel A 2008 Récupération dénergie et contrôle vibratoire par éléments piézoélectriques suivant une approche non linéaire PhD Thesis Université de Savoie

[34] Lallart M 2008 Amélioration de la conversion électroactive de matériaux piézoélectriques et pyroélectriques pour le contrôle vibratoire et la récupération d'énergie-Application au contrôle de santé structurale auto-alimenté PhD Thesis INSA de Lyon

[35] Std I 1988 IEEE standard on piezoelectricity (https://doi.org/ 10.1109/IEEESTD.1988.79638)

[36] Guyomar D, Badel A, Lefeuvre E and Richard C 2005 Toward energy harvesting using active materials and conversion improvement by nonlinear processing IEEE Trans. Ultrason. Ferroelectr. Freq. Control 52 584-95

[37] Yi K, Collet M, Ichchou M and Li L 2016 Flexural waves focusing through shunted piezoelectric patches Smart Mater. Struct. 25075007 